\title{
The effect of tartary buckwheat protein on the growth of probiotics
}

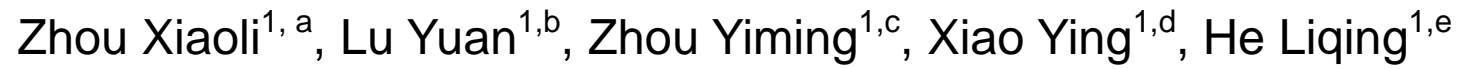 \\ 1Shanghai Institute of Technology, Shanghai China

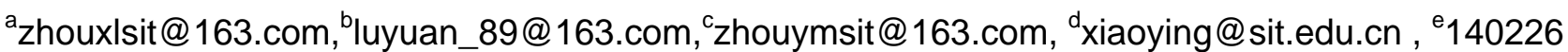 \\ 7181@qq.com
}

Keywords: probiotics; prebiotics; bile acid salts; Lactobacilli; Bifidobacteria

\begin{abstract}
Tartary buckwheat is a kind of food and medicinal material and it contains abundant protein. Tartary buckwheat protein has the characteristics of indigestibility and other physiological activities The main purpose of this paper is research the prebiotic effects of protein extract of tartary buckwheat on probiotics in vivo experiments. Thus, the growth of probiotics in bile acids salts and the growth of probiotics in the presence of tartary buckwheat protein as well as the effect of tartary buckwheat protein on probiotics bile acid salts tolerance were carried out in this paper. The results showed that tartary buckwheat protein could promote the growth of L.casei LC2W and B.longum BD 400 and tartary buckwheat protein could increase the SC and SGC tolerance of L.casei LC2W and B.longum BD 400, thereby, increasing the growth rate of the above two strains of probiotics, and compared with the control group the OD values were increased by $77.51 \%, 73.95 \%$ and $70.95 \%, 70.37 \%$. Thus it can be seen that tartary buckwheat protein may be used as a kind of growth factor for probiotics. This study provides a theoretical basis for the application of buckwheat and buckwheat protein in food.
\end{abstract}

\section{Introduction}

The genus Fagopyrum (buckwheat) is a part of the family of Polygonaceae, which has approximately fifteen species all over the world[1]. But only tartary buckwheat (Fagopyrum tataricum) and common buckwheat (Fagopyrum esculentum) are used as food and medicinal material[2]. Currently, the importance and research interest concern of tartary buckwheat in human health has become increasingly obvious. Now, the demand for tartary buckwheat is increasing in the food nutrition, pharmaceutical and cosmetic industries, owing to its high nutritional and medical value[3].

It is well known that tartary buckwheat has comparatively more functional phytochemicals and nutrient compounds than common buckwheat, such as protein, flavonoids, starch, dietary fiber and other nutrients [4,5]. Buckwheat protein contains a well-balanced amino acid proportion and is rich in tryptophan and lysine as well as has a high biological value[6,7]. Until 1995, researchers provided the first evidence about the hypocholest- erolemic effect of tartary buckwheat protein in cholesterol-fed rats, which was far better than that of soy protein[8]. Since then, the physiological properties of tartaty buckwheat protein have been researched and proved that buckwheat protein extract possessed several physiology functions, including hypocholesterolemic, lowering cholesterol and so on[9].

There are 400-500 kinds of intestinal bacteria in the intestinal tract of healthy adult. The number of intestinal bacteria are as high as $10^{11-12} \mathrm{CFU} / \mathrm{g}[10]$. The intestinal flora mainly consists of probiotics and non probiotics (pathogenic bacteria). Lactobacilli and Bifidobacteria are the two of most representative probiotics. They play an important role in maintaining the normal microbial flora in the intestinal tract. The acetic acid and hydrogen peroxide produced by probiotics has the function of reducing the intestinal $\mathrm{pH}$ and inhibiting the proliferation of the bacteria, so the formation of the potential carcinogens in the colon is reduced. The research shows that they are useful to the human body, such as the treatment of food allergies, diarrhea, delaying human aging, regulating blood lipids and other functions and they are widely used in all kinds of food as food additives. The intaking of prebiotics have the effect of regulatory on the intestinal flora, and can be 
beneficial to promote the balance of intestinal flora. However, The conditions for the beneficial effects of Lactobacillus and Bifidobacteria on the human condition are to be able to survive in the extreme gastrointestinal tract[11].

To the extent that prebiotics, it mainly includes inulin-fructose such as fructo-oligosaccharides, inulin, galacto-oligosaccharides and lactulose[12]. Further- more, protein, peptide and unsaturated fatty acid also can promote the growth of probiotics[13]. However, relatively few experiments have been conducted to determine the prebiotic effects of protein in humans and very litter specific information is available regarding tartary buckwheat protein regulate probiotics. Even the research about buckwheat protein,probiotics and bile salt environment has not been reported. Thus, the objective of present studies was to evaluate the prebiotic effects of protein extract of tartary buckwheat on probiotics in vivo experiments.

\section{Materials and methods}

\section{Chemicals and reagents}

Black Feng No. 1 buckwheat, Zuoyun County, Shanxi Province; Sodium Cholate(SC), sodium deoxidation cholate(SDC), Sodium Taurocholate(STC), Sodium Glycyl Cholate(SGC), Shanghai Boman Biological Technology Co.,Ltd.; Double distilled water was used throughout; All other reagents were of analytical reagent(AR)grade, Sinopharm chemicals ( Shanghai ) Co., Ltd;

\section{Bacterial strains}

B.longum BD 0400 and L.casei LC2W(CGMCC), Shanghai Guangming dairy R \& D Center.

\section{Growth media}

Bifidobacterium BS Medium for Bifidobacterium and MRS broth medium for Lactobacillus, Qingdao hope bio-Technology Co., Ltd.

\section{Preparation of tartary buckwheat protein}

tartary buckwheat seed, smash and sift, degrease, PBS dissolve (W/V=1:10), agitate ( $2 \mathrm{~h})$, centrifuge(4, C 4000r/min , 30min), add ammonium sulfate(W\%=40\%), agitate ( $1 \mathrm{~h}$ ) , centrifuge (4, C 10000 $/ \mathrm{min}, 30 \mathrm{~min})$, add ammonium sulfate(W\%=80\%), agitate ( $1 \mathrm{~h}$ ) , centrifuge (4, C10000r/min, 30min), dissolute with double distilled water, dialyze and freezedrying.

\section{Growth of probiotics in bile acids salts}

Bacterial counts for each experiments was achieved using the successive dilution method, $2 \times 10^{3}$ $\mathrm{CFU} / \mathrm{mL}$ was used as the starting inoculum concentration for all experiments. To be short, Bifidobacterium BS Medium and MRS broth medium containing $2 \times 10$ 3CFU/mL cultures was introduced into 96-well sterile microtiter plates (Beyotime, China). Only added equal volumes above medium as control and bile acids salts at final concentrations (1-6 mM) reflect those found in human colon. The 96 -well plates were incubated in an anaerobic conditions $\left(5 \% \mathrm{CO}_{2}, 5 \% \mathrm{H}_{2}\right.$ and the rest is $\mathrm{N}_{2}$ ) of growth at $37^{\circ} \mathrm{C}$ for $32 \mathrm{~h}$. The optical density(OD) value of bacterial growths were monitored by every $8 \mathrm{~h}$ at $600 \mathrm{~nm}(\mathrm{OD} 600)$ using the multiplate reader(Infinite M200PRO, TECAN ).

\section{Growth of probiotics in the presence of tartary buckwheat protein}

To determining the growth effect of tartary buckwheat protein on Lactobacillus and Bifidobacterium strains. Experiments were carried out as follows: Basal medium was respectively supplemented with $1,3,5 \mathrm{mg} / \mathrm{mL}$ tartary buckwheat protein and casein(casein as control group) as well as Lactobacillus and Bifidobacterium strains. The 96-well plates were incubated in an anaerobic conditions(5\%CO2,5\%H2 and the rest is $\mathrm{N} 2)$ of growth at $37^{\circ} \mathrm{C}$ for $32 \mathrm{~h}$ and $\mathrm{OD} 600$ was measured every $4 \mathrm{~h}$.

\section{Effect of tartary buckwheat protein on probiotics tolerance bile acid salt}

To determine the effect of tartary buckwheat protein on probiotics tolerance to bile acid, the basal medium described above was supplemented with $5 \mathrm{mg} / \mathrm{mL}$ tartary buckwheat protein, casein and $6 \mathrm{mM}$ Sodium Cholate(SC), Sodium Glycyl Cholate(SGC). The 96-well plates were incubated in an anaerobic conditions $\left(5 \% \mathrm{CO}_{2}, 5 \% \mathrm{H}_{2}\right.$ and the rest is $\left.\mathrm{N}_{2}\right)$ of growth at $37^{\circ} \mathrm{C}$ for $32 \mathrm{~h}$ and $\mathrm{OD}$ 
600 was measured every $4 \mathrm{~h}$.

\section{Statistical analysis}

All data were expressed by Means + SD. ANOVA (LSD test) were used to examine the significance of the data with SPSS 20.0 software, and p values $<0.05$ were considered to be statistically significant. All measurements were performed in 5 wells.

\section{Results and Discussion}

\section{Growth of the probiotics in the presence of bile acids salts}

However, due to the changeable physicochemical properties of $\mathrm{pKa}$ and LogP, the bile acid salts solubility in media varies significantly. Consequently, it was not possible to expose the probiotics to the same range of concentrations for all the bile acids (Table 1). The highest soluble concentration of SDC $(0.5 \mathrm{mM})$ did not inhibit the growth of two strains probiotics(Table 1). The inhibition of STC is similar to SDC, namely, the highest concentration of STC $(6 \mathrm{mM})$ had not inhibition for probiotics (Table 1). Compared with $\mathrm{SC}(\geq 1.5 \mathrm{mM})$ inhibiting the growth of all probiotics, when the concentration of SC reaches to $6 \mathrm{mM}$, the minimal growth is demonstrated(Table 1). Under the concentration of STC great than $1.25 \mathrm{mM}$, the growth of L.casei LC2W and B.longum BD 400 was inhibited.

The results of paper is consistent with Smet ID, The growth of probiotics can inhibited by bile acid salts and the survival rate of probiotics rely on the concentration and type of bile acid salts[14]. Therefore, selecting the choice of experimental $6 \mathrm{~mm}$ sodium cholate(SC) and sodium glycyl cholate(SGC)for further research the effect of tartary buckwheat protein on probiotics tolerance to bile salts.

Table1 The effect of different bile salts on the growth of two strains probiotics

\begin{tabular}{cccc}
\hline & Bile acids salts $(\mathrm{mM})$ & L.casei LC2W & B.longum BD 400 \\
\hline \multirow{3}{*}{ SDC } & 0.5 & +++ & ++ \\
& 0.25 & +++ & +++ \\
& 0.125 & +++ & +++ \\
\cline { 2 - 4 } SC & 6 & + & ++ \\
& 3 & ++ & ++ \\
\cline { 2 - 4 } SGC & 1.5 & ++ & + \\
& 6 & + & ++ \\
& 3 & ++ & ++ \\
STC & 1.5 & ++ & +++ \\
& 6 & +++ & +++ \\
\hline
\end{tabular}

+++: Comparable with growth in no bile stress;++:Less growth;+:Minimal growth

\section{Growth of probiotics in the presence of tartary buckwheat protein}

Growth of the probiotics in the presence of tartary buckwheat protein substrates was compared to growth in the basal medium (negative control) and to the presence of casein as the growth substrate (positive control). Growth of L.casei LC2W and B.longum BD 400 (Fig. 1) in the presence of tartary buckwheat protein was significantly ( $<0.05$ )higher after $12 \mathrm{~h}$ than growth in the basal medium and higher after $20 \mathrm{~h}$ comparable to growth in the presence of casein (Fig. 1). The OD600 value of two strains probiotics showed decreasing tendency gradually in the presence of casein after $16 \mathrm{~h}$. With the increase of substrate concentration, the promoting growth effect also improved. In addition, the promoting effect of same concentration tartary buckwheat protein on L.casei greater B.longum. Although the mechanism of selective substrate utilisation is not clearly understood, the presence or absence of specific transport and hydrolysis systems for particular substrate is a widely accepted view[15]. 


\section{Effect of tartary buckwheat protein on probiotics tolerance bile acid salt}

The results show that the ability of L. casei LC2W and B. longum BD 400 to withstand bile acids salts under the different sbustrates(Fig.2). the results shows that tartary buckwheat protein could increase the SC and SGC tolerance of L. casei LC2W and B. longum BD 400,thereby,incresing the growth rate of the above two strains of probiotics, and compared with the control group the OD values were increased by $77.51 \%, 73.95 \%$ and $70.95 \%, 70.37 \%$. Effect of tartary buckwheat protein of the two strains of probiotics tolerance bile acid salts were significantly higher than those of casein $(\mathrm{P}<0.05)$,this may be due to part of bile salts were adsorbed by tartary buckwheat protein to reduce the inhibition of bile acid salts. Experiments show that, tartary buckwheat protein can significantly improve probiobics growth under bile acid salts stress. Similarly, Saarela showed that lactulose might improve the bile tolerance of lactic acid bacteria[16].
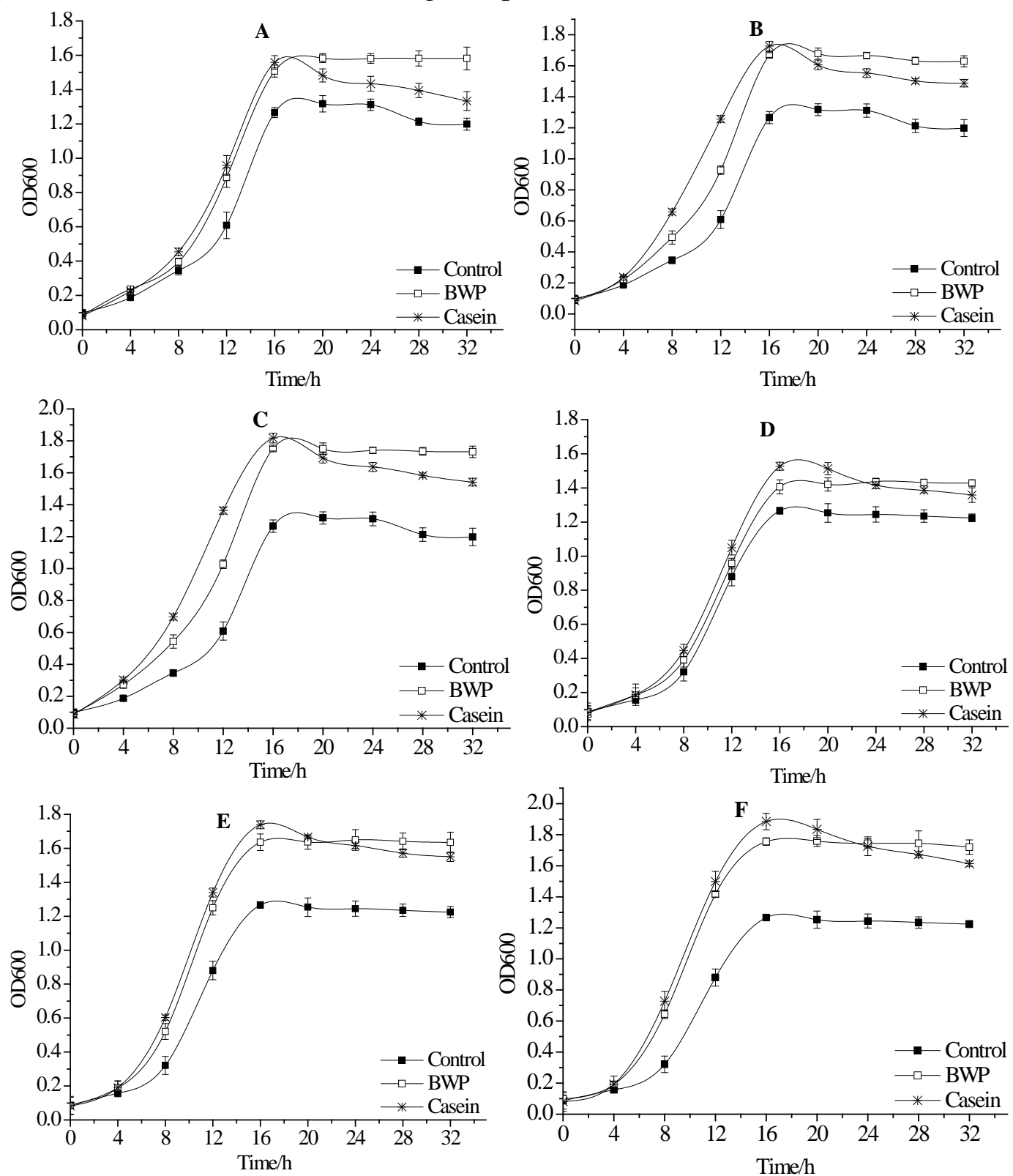

Fig.1 The effect of tartary buckwheat protein on the growth of two strains probiotics. Lactobacillus(A-C) and Bifidobacterium(D-F) exposed to tartary buckwheat protein A D:1mg/mL;B $\mathrm{E}: 2.5 \mathrm{mg} / \mathrm{mL} ; \mathrm{C} F: 5 \mathrm{mg} / \mathrm{m}$ :. Each point represents the mean and $\pm \mathrm{SD}$ of at least five determinations 

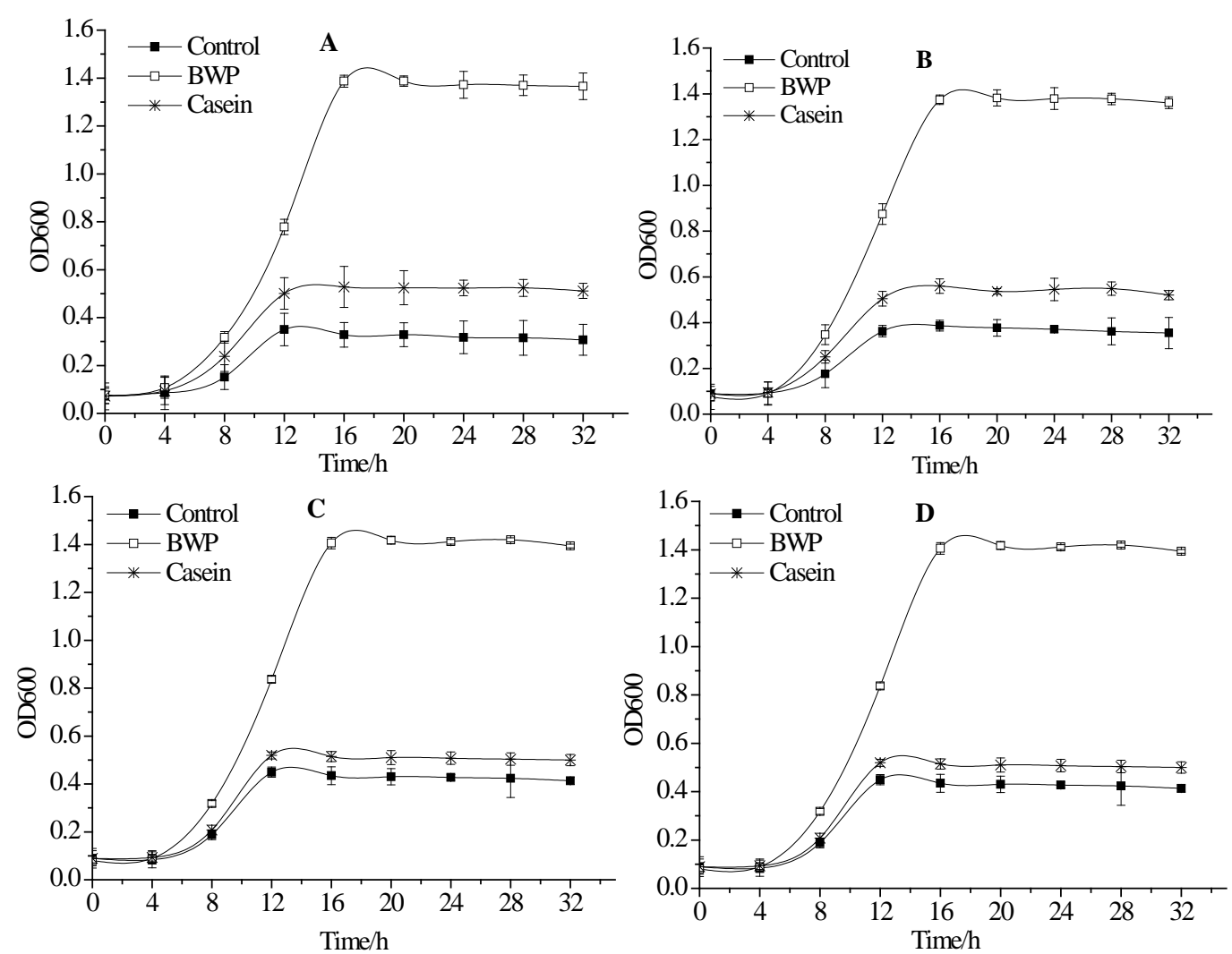

Fig.2 Growth of L.casei LC2W and B.longum BD 400 in bile acid salts stress following tartary buckwheat protein.L. casei LC2W(A-B) and B. longum BD400(C-D) exposed to casein or 5mg/mL tartary buckwheat protein, $6 \mathrm{mM}$ Sodium Cholate (A and C), $6 \mathrm{mM}$ Sodium Glycyl Cholate(B and $\mathrm{D})$.each point represents the mean and $\pm \mathrm{SD}$ of at least five determinations

\section{Conclusions}

In order to research the beneficial effect of tartary buckwheat protein on probiotics, We have studied from three aspects. There was no evidence that tartary buckwheat protein could promote the growth of L.casei LC2W and B.longum BD 400 in these anaerobic experiments. On the other hand, tartary buckwheat protein could improve the bile acid salts tolerance of probiotics. The reason as follow: firstly, it could be one of the important sources of probiotics energy in the colon in vivo[17]. Secondly, it could also heighten probiotics growth by decreasing the intestinal tract $\mathrm{pH}$ to an optimum level and a factor affected by the physicochemical properties of the bile acid salts. Finally, the high concentrations of substrate may decrease the solubility of bile acid salts which may decrease toxicity. In a word, tartary buckwheat protein is beneficial to human health.

\section{Acknowledgements}

This study was financed by the National Nature Science Foundation of China (No.31371761 to Zhou XL). The anthors thank Xiaoli Zhou and Yiming Zhou and Ying Xiao for their assistance in some experiments.

\section{References}

[1] Tahir, I., Farooq, S. Grain composition in some buckwheat cultivars (Fagopyrum spp.) with particular reference to protein fraction. Plant Foods for Human Nutrition, 1985, 35(2): 153-158.

[2] G. Bonafaccia, M. Marocchini, I. Kreft, Composition and technological properties of the flour and bran from common and tartary buckwheat. Food Chemistry, 2003, 80(1): 9-15.

[3] Wang X, Feng B, Xu Z, et al. Identification and characterization of granule bound starch 
synthase I(GBSSI) gene of tartary buckwheat (Fagopyrum tataricum Gaertn.). Gene, 2014, 534(2): 229-235.

[4] Bonafaccia G, Marocchini M, Kreft I.. Composition and technological properties of the flour and bran from common and tartary buckwheat. Food Chemisty, 2003, 80: 9-15.

[5] Kitabayashi H, Ujihara A, Hirose T, et al.On the genotypic differences for rutin content in tartary buckwheat, Fagopyrum tataricum Gaertn. Breeding Science, 1995，45: 189-194.

[6] Pomeranz Yeshajahu, Lorenz K. Buckwheat: structure, composition, and utilization. C R C Critical Reviews in Food Science and Nutrition, 1983, 19(3): 213-258

[7] Yeshajahu P, George S, Robbins. Amino Acid Composition of Buckwheat.Journal of agricultural and food chemistry. 1972, 20 (4): 922-922.

[8] Jun K, Iwao S, Misao N, Hypocholesterolemic effect of buckwheat protein extract in rats fed cholesterol-enriched diet. Nutrition Research, 1995, 15(5): 691-698.

[9] Jun K, Iwao S, Misao N, et al. Consumption of buckwheat protein lowers plasma cholesterol and raises fecal neutral sterols in cholesterol-fed rats because of its low digestibility. Journal of Nutrition, 1997, 127(7):1395-1400.

[10]Kedia G, Vazquez JA. In vitro fermentation of oat bran obtained by debranning with a mixed culture of human fecal bacteria. Current Microbiology, 2009, 58(4): 338-342.

[11] Oluwakemi OA, Olivia C, Winston A. Synbiotics: the impact of potential prebiotics inulin, lactulose and lactobionic acid on the survival and growth of lactobacilli probiotics. Journal of Functional Foods, 2014, 10:75-84.

[12]Gibson, GR. Dietary modulation of the human colonic microbiota: updating the concept of prebiotics. Nutrition Research Reviews, 2004, 17:259-275.

[13]Janer C. Pelaez, T. Requena. Caseinomacropeptide and whey protein concentrate enhance Bifidobacterium lactis growth in milk, Food Chemistry, 2004(86): 263-267.

[14] Smet ID, Hoorde LV, Woestyne MV, et al. Significance of bile salt hydrolyticactivities of lactobacilli.Journal of Applied Microbiology, 1995, 79 (3): 292-301.

[15]Kaplan H, Hutkins RW. Metabolism of fructooligosaccharides by Lactobacillus paracasei 1195. Applied and Environmental Microbiology, 2003， 69: 2217-2222.

[16] Saarela M, Hallamaa K, Mattila-Sandholm T, et al. The effect of lactose derivatives lactulose, lactitol and lactobionic acid on the functional and technological properties of potentially probiotic Lactobacillus strains. International Dairy Journal， 2003， 13: 291-302.

[17] Schneeman BO. Fiber, inulin and oligofructose: Similarities and differences. The Journal of Nutrition, 1999, 129: 1424-1427. 